\title{
The Split Common Fixed Point Problem for Total Asymptotically Strictly Pseudocontractive Mappings
}

\author{
S. S. Chang, ${ }^{1}$ L. Wang, ${ }^{1}$ Y. K. Tang, ${ }^{1}$ and L. Yang ${ }^{2}$ \\ ${ }^{1}$ Department of Mathematics, College of Statistics and Mathematics, Yunnan University of Finance and \\ Economics, Kunming, Yunnan 650221, China \\ ${ }^{2}$ Department of Mathematics, South West University of Science and Technology, Mianyang, \\ Sichuan 621010, China
}

Correspondence should be addressed to S. S. Chang, changss@yahoo.cn

Received 4 September 2011; Accepted 21 October 2011

Academic Editor: Yonghong Yao

Copyright (C) 2012 S. S. Chang et al. This is an open access article distributed under the Creative Commons Attribution License, which permits unrestricted use, distribution, and reproduction in any medium, provided the original work is properly cited.

The purpose of this paper is to propose an algorithm for solving the split common fixed point problems for total asymptotically strictly pseudocontractive mappings in infinite-dimensional Hilbert spaces. The results presented in the paper improve and extend some recent results of Moudafi (2011 and 2010), Xu (2010 and 2006), Censor and Segal (2009), Censor et al. (2005), Masad and Reich (2007), Censor et al. (2007), Yang (2004), and others.

\section{Introduction}

Throughout this paper, we always assume that $H_{1}, H_{2}$ are real Hilbert spaces, " $\rightarrow$, - " denote by strong and weak convergence, respectively, and $F(T)$ is the fixed point set of a mapping $T$.

The split common fixed point problem (SCFP) is a generalization of the split feasibility problem (SEP) and the convex feasibility problem (CFP). It is worth mentioning that SFP in finite-dimensional spaces was first introduced by Censor and Elfving [1] for modeling inverse problems which arise from phase retrievals and in medical image reconstruction [2]. Recently, it has been found that the SFP can also be used in various disciplines such as image restoration, computer tomograph, and radiation therapy treatment planning [3-5].

SEP in an infinite-dimensional Hilbert space can be found in [2, 4, 6-8]. Moreover the convex feasibility formalism is at the core of the modeling of many inverse problems and has been used to model significant real-world problems. 
The split common fixed point problems for a class of quasi-nonexpansive mappings and demicontractive mappings in the setting of Hilbert space were first introduced and studied by Moudafi $[9,10]$.

The purpose of this paper is to introduce and study the following split common fixed point problem for a more general class of total asymptotically strict pseudocontraction (SCFP) in the framework of an infinite-dimensional Hilbert spaces which contains the quasi-nonexpansive mappings and the demicontractive mappings as its special cases:

$$
\text { find } x^{*} \in C \text { such that } A x^{*} \in Q \text {, }
$$

where $A: H_{1} \rightarrow H_{2}$ is a bounded linear operator, $S: H_{1} \rightarrow H_{1}$ and $T: H_{2} \rightarrow H_{2}$ are mappings $C:=F(S)$, and $Q:=F(T)$. In the sequel we use $\Gamma$ to denote the set of solutions of (SCFP), that is,

$$
\Gamma=\{x \in C, A x \in Q\}
$$

\section{Preliminaries}

We first recall some definitions, notations, and conclusions which will be needed in proving our main results.

Let $E$ be a Banach space. A mapping $T: E \rightarrow E$ is said to be demiclosed at origin, if for any sequence $\left\{x_{n}\right\} \subset E$ with $x_{n} \rightarrow x^{*}$ and $\left\|(I-T) x_{n}\right\| \rightarrow 0, x^{*}=T x^{*}$. $x_{n} \rightarrow x^{*}$,

A Banach space $E$ is said to have the Opial property, if for any sequence $\left\{x_{n}\right\}$ with

$$
\liminf _{n \rightarrow \infty}\left\|x_{n}-x^{*}\right\|<\liminf _{n \rightarrow \infty}\left\|x_{n}-y\right\|, \quad \forall y \in E \text { with } y \neq x^{*}
$$

Remark 2.1. It is well known that each Hilbert space possesses the Opial property.

Definition 2.2. Let $H$ be a real Hilbert space, and let $K$ be nonempty and closed convex subset of $H$.

(1) A mapping $G: K \rightarrow K$ is said to be $\left(\gamma,\left\{\mu_{n}\right\},\left\{\xi_{n}\right\}, \phi\right)$-totally asymptotically strictly pseudocontractive, if there exist a constant $\gamma \in[0,1)$ and sequences $\left\{\mu_{n}\right\} \subset[0, \infty)$ and $\left\{\xi_{n}\right\} \subset[0, \infty)$ with $\mu_{n} \rightarrow 0$ and $\xi_{n} \rightarrow 0$ such that for all $x, y \in K$

$$
\begin{aligned}
\left\|G^{n} x-G^{n} y\right\|^{2} \leq & \|x-y\|^{2}+\gamma\left\|\left(I-G^{n}\right) x-\left(I-G^{n}\right) y\right\|^{2} \\
& +\mu_{n} \phi(\|x-y\|)+\xi_{n}, \quad \forall n \geq 1,
\end{aligned}
$$

where $\phi:[0, \infty) \rightarrow[0, \infty)$ is a continuous and strictly increasing function with $\phi(0)=0$.

(2) A mapping $G: K \rightarrow K$ is said to be $\left(\gamma,\left\{k_{n}\right\}\right)$-asymptotically strictly pseudocontractive, if there exist a constant $\gamma \in[0,1)$ and a sequence $\left\{k_{n}\right\} \subset[1, \infty)$ with $k_{n} \rightarrow 1$ such that

$$
\left\|G^{n} x-G^{n} y\right\|^{2} \leq k_{n}\|x-y\|^{2}+\gamma\left\|\left(I-G^{n}\right) x-\left(I-G^{n}\right) y\right\|^{2}, \quad \forall x, y \in K .
$$


(3) Especially, if there exists $\gamma \in[0,1)$ such that

$$
\|G x-G y\|^{2} \leq\|x-y\|^{2}+\gamma\|(I-G) x-(I-G) y\|^{2}, \quad \forall x, y \in K,
$$

then $G: K \rightarrow K$ is called a $\gamma$-strictly pseudocontractive mapping.

(4) A mapping $G: K \rightarrow K$ is said to be uniformly L-Lipschitzian, if there exists a constant $L>0$, such that

$$
\left\|G^{n} x-G^{n} y\right\| \leq L\|x-y\|, \quad \forall x, y \in K, n \geq 1 .
$$

(5) A mapping $G: K \rightarrow K$ is said to be semicompact, if for any bounded sequence $\left\{x_{n}\right\} \subset K$ with $\lim _{n \rightarrow \infty}\left\|x_{n}-G x_{n}\right\|=0$, there exists a subsequence $\left\{x_{n_{i}}\right\} \subset\left\{x_{n}\right\}$ such that $\left\{x_{n_{i}}\right\}$ converges strongly to some point $x^{*} \in K$.

Remark 2.3. If $\phi(\lambda)=\lambda^{2}, \lambda \geq 0$, and $\xi_{n}=0$, then a $\left(\gamma,\left\{\mu_{n}\right\},\left\{\xi_{n}\right\}, \phi\right)$-total asymptotically strictly pseudocontractive mapping is an $\left(\gamma,\left\{k_{n}\right\}\right)$-asymptotically strict pseudocontractive mapping, where $\left\{k_{n}=1+\mu_{n}\right\}$.

Proposition 2.4. Let $G: K \rightarrow K$ be $a\left(\gamma,\left\{\mu_{n}\right\},\left\{\xi_{n}\right\}, \phi\right)$-total asymptotically strictly pseudocontractive mapping. If $F(G) \neq \emptyset$, then for each $q \in F(G)$ and for each $x \in K$, the following inequalities hold and they are equivalent:

$$
\begin{gathered}
\left\|G^{n} x-q\right\|^{2} \leq\|x-q\|^{2}+\gamma\left\|x-G^{n} x\right\|^{2}+\mu_{n} \phi(\|x-q\|)+\xi_{n} \\
\left\langle x-G^{n} x, x-q\right\rangle \geq \frac{1-\gamma}{2}\left\|x-G^{n} x\right\|^{2}-\frac{\mu_{n}}{2} \phi(\|x-q\|)-\frac{\xi_{n}}{2} \\
\left\langle x-G^{n} x, q-G^{n} x\right\rangle \leq \frac{\gamma+1}{2}\left\|G^{n} x-x\right\|^{2}+\frac{\mu_{n}}{2} \phi(\|x-q\|)+\frac{\xi_{n}}{2} .
\end{gathered}
$$

Proof. (I) Inequality (2.6) can be obtained from (2.2) immediately.

(II) $(2.6) \Leftrightarrow(2.7)$ In fact, since

$$
\begin{aligned}
\left\|G^{n} x-q\right\|^{2} & =\left\|G^{n} x-x+x-q\right\|^{2} \\
& =\left\|G^{n} x-x\right\|^{2}+\|x-q\|^{2}+2\left\langle G^{n} x-x, x-q\right\rangle, \quad \forall x \in K, q \in F(G),
\end{aligned}
$$

from (2.6) we have that

$$
\begin{gathered}
\left\|G^{n} x-x\right\|^{2}+\|x-q\|^{2}+2\left\langle G^{n} x-x, x-q\right\rangle \\
\leq\|x-q\|^{2}+\gamma\left\|x-G^{n} x\right\|^{2} \\
+\mu_{n} \phi(\|x-q\|)+\xi_{n} .
\end{gathered}
$$

Simplifying it, the inequality (2.7) is obtained. 
Conversely, from (2.7) the inequality (2.6) can be obtained immediately.

(III) $(2.7) \Leftrightarrow(2.8)$ In fact, since

$$
\begin{aligned}
\left\langle x-G^{n} x, x-q\right\rangle & =\left\langle x-G^{n} x, x-G^{n} x+G^{n} x-q\right\rangle \\
& =\left\|x-G^{n} x\right\|^{2}+\left\langle x-G^{n} x, G^{n} x-q\right\rangle,
\end{aligned}
$$

it follows from (2.7) that

$$
\left\|x-G^{n} x\right\|^{2}+\left\langle x-G^{n} x, G^{n} x-q\right\rangle \geq \frac{1-\gamma}{2}\left\|x-G^{n} x\right\|^{2}-\frac{\mu_{n}}{2} \phi(\|x-q\|)-\frac{\xi_{n}}{2} .
$$

Simplifying it, the inequality (2.8) is obtained.

Conversely, the inequality (2.7) can be obtained from (2.8) immediately.

This completes the proof of Proposition 2.4.

Lemma 2.5 (see [11]). Let $\left\{a_{n}\right\},\left\{b_{n}\right\}$, and $\left\{\delta_{n}\right\}$ be sequences of nonnegative real numbers satisfying

$$
a_{n+1} \leq\left(1+\delta_{n}\right) a_{n}+b_{n}, \quad \forall n \geq 1
$$

If $\sum_{i=1}^{\infty} \delta_{n}<\infty$ and $\sum_{i=1}^{\infty} b_{n}<\infty$, then the limit $\lim _{n \rightarrow \infty} a_{n}$ exists.

Lemma 2.6 (see [12]). Let $H$ be a real Hilbert space. If $\left\{x_{n}\right\}$ is a sequence in $H$ weakly convergent to $z$, then

$$
\underset{n \rightarrow \infty}{\limsup }\left\|x_{n}-y\right\|^{2}=\limsup _{n \rightarrow \infty}\left\|x_{n}-z\right\|^{2}+\|z-y\|^{2}, \quad \forall y \in H
$$

Proposition 2.7. Let $H$ be a real Hilbert space and let $T: H \rightarrow H$ be a uniformly L-Lipschitzian and $\gamma,\left\{\mu_{n}\right\},\left\{\xi_{n}\right\}, \phi$-total asymptotically strictly pseudocontractive mapping. Then the demiclosedness principle holds for $T$ in the sense that if $\left\{x_{n}\right\}$ is a sequence in $H$ such that $x_{n} \rightarrow x^{*}$, and $\limsup _{m \rightarrow \infty} \limsup _{n \rightarrow \infty}\left\|x_{n}-T^{m} x_{n}\right\|=0$, then $(I-T) x^{*}=0$. In particular, if $x_{n} \rightarrow x^{*}$, and $\left\|(I-T) x_{n}\right\| \rightarrow 0$, then $(I-T) x^{*}=0$, that is, $T$ is demiclosed at origin.

Proof. Since $\left\{x_{n}\right\}$ is bounded, we can define a function $f$ on $H$ by

$$
f(x)=\limsup _{n \rightarrow \infty}\left\|x_{n}-x\right\|^{2}, \quad \forall x \in H .
$$

Since $x_{n} \rightarrow x^{*}$, it follows from Lemma 2.6 that

$$
f(x)=f\left(x^{*}\right)+\left\|x-x^{*}\right\|^{2}, \quad \forall x \in H .
$$

In particular, for each $m \geq 1$,

$$
f\left(T^{m} x^{*}\right)=f\left(x^{*}\right)+\left\|T^{m} x^{*}-x^{*}\right\|^{2} .
$$


On the other hand, since $T$ is a $\left(\gamma,\left\{\mu_{n}\right\},\left\{\xi_{n}\right\}, \phi\right)$-total asymptotically strictly pseudocontraction mapping, we get

$$
\begin{aligned}
f\left(T^{m} x^{*}\right)= & \limsup _{n \rightarrow \infty}\left\|x_{n}-T^{m} x^{*}\right\|^{2} \\
= & \limsup _{n \rightarrow \infty}\left\|x_{n}-T^{m} x_{n}+T^{m} x_{n}-T^{m} x^{*}\right\|^{2} \\
= & \limsup _{n \rightarrow \infty}\left(\left\|x_{n}-T^{m} x_{n}\right\|^{2}+2\left\langle x_{n}-T^{m} x_{n}, T^{m} x_{n}-T^{m} x^{*}\right\rangle+\left\|T^{m} x_{n}-T^{m} x^{*}\right\|^{2}\right) \\
\leq & \limsup _{n \rightarrow \infty}\left\|x_{n}-T^{m} x_{n}\right\|\left(\left\|x_{n}-T^{m} x_{n}\right\|+2 L\left\|x_{n}-x^{*}\right\|\right) \\
& +\limsup _{n \rightarrow \infty}\left(\left\|x_{n}-x^{*}\right\|^{2}+\gamma\left\|x_{n}-T^{m} x_{n}-\left(x^{*}-T^{m} x^{*}\right)\right\|^{2}+\mu_{m} \phi\left(\left\|x_{n}-x^{*}\right\|\right)+\xi_{m}\right) .
\end{aligned}
$$

Taking $\lim \sup _{m \rightarrow \infty}$ on both sides and observing the facts that $\lim _{m \rightarrow \infty} \mu_{m}=0, \lim _{m \rightarrow \infty} \xi_{m}=0$, and $\lim \sup _{m \rightarrow \infty} \limsup _{n \rightarrow \infty}\left\|x_{n}-T^{m} x_{n}\right\|=0$, we derive that

$$
\begin{aligned}
\limsup _{m \rightarrow \infty} f\left(T^{m} x^{*}\right) & \leq \underset{m \rightarrow \infty}{\limsup }\left\|x_{n}-x^{*}\right\|^{2}+\gamma \underset{m \rightarrow \infty}{\limsup }\left\|x^{*}-T^{m} x^{*}\right\|^{2} \\
& =f\left(x^{*}\right)+\underset{m \rightarrow \infty}{\limsup }\left\|x^{*}-T^{m} x^{*}\right\|^{2} .
\end{aligned}
$$

On the other hand, it follows from (2.17) that

$$
\limsup _{m \rightarrow \infty} f\left(T^{m} x^{*}\right)=f\left(x^{*}\right)+\limsup _{m \rightarrow \infty}\left\|x^{*}-T^{m} x^{*}\right\|^{2}
$$

Since $\kappa<1$, this together with (2.19) shows that $\limsup _{m \rightarrow \infty}\left\|x^{*}-T^{m} x^{*}\right\|^{2}=0$. That is, $\lim _{m \rightarrow \infty} T^{m} x^{*}=x^{*}$; hence $T x^{*}=x^{*}$.

\section{Split Common Fixed Point Problem}

For solving the split common fixed point problem (1.1), let us assume that the following conditions are satisfied.

(1) $H_{1}$ and $H_{2}$ are two real Hilbert spaces, and $A: H_{1} \rightarrow H_{2}$ is a bounded linear operator.

(2) $S: H_{1} \rightarrow H_{1}$ is a uniformly $L$-Lipschitzian and $\left(\beta,\left\{\mu_{n}^{(1)}\right\},\left\{\xi_{n}^{(1)}\right\}, \phi_{1}\right)$-total asymptotically strictly pseudocontractive mapping and $T: H_{2} \rightarrow H_{2}$ is a uniformly $\tilde{L}$-Lipschitzian 
and $\left(\kappa,\left\{\mu_{n}^{(2)}\right\},\left\{\xi_{n}^{(2)}\right\}, \phi_{2}\right)$-total asymptotically strictly pseudocontractive mapping satisfying the following conditions:

(i) $C:=F(S) \neq \emptyset, Q:=F(T) \neq \emptyset$;

(ii) $\mu_{n}=\max \left\{\mu_{n}^{(1)}, \mu_{n}^{(2)}\right\}, \xi_{n}=\max \left\{\xi_{n}^{(1)}, \xi_{n}^{(2)}\right\}, n \geq 1$, and $\sum_{n=1}^{\infty} \mu_{n}<\infty, \sum_{n=1}^{\infty} \xi_{n}<\infty$;

(iii) $\phi=\max \left\{\phi_{1}, \phi_{2}\right\}$ and there exist two positive constants $M$ and $M^{*}$ such that $\phi(\lambda) \leq$ $M^{*} \lambda^{2}$ for all $\lambda \geq M$.

We are now in a position to give the following result.

Theorem 3.1. Let $H_{1}, H_{2}, A, S, T, C, Q, \beta, \kappa, L, \widetilde{L},\left\{\mu_{n}\right\},\left\{\xi_{n}\right\}$, and $\phi$ be the same as mentiond before. Let $\left\{x_{n}\right\}$ be the sequence generated by

$$
\begin{gathered}
x_{1} \in H_{1} \text { chosen arbitrarily, } \\
x_{n+1}=\left(1-\alpha_{n}\right) u_{n}+\alpha_{n} S^{n}\left(u_{n}\right), \\
u_{n}=x_{n}+\gamma A^{*}\left(T^{n}-I\right) A x_{n}, \quad \forall n \geq 1,
\end{gathered}
$$

where $\left\{\alpha_{n}\right\}$ is a sequence in $[0,1]$ and $\gamma>0$ is a constant satisfying the following conditions:

(iv) $\alpha_{n} \in(\delta, 1-\beta)$, for all $n \geq 1$ and $\gamma \in\left(0,(1-\kappa) /\|A\|^{2}\right)$, where $\delta \in(0,1-\beta)$ is a positive constant.

(I) If $\Gamma \neq \emptyset$ (where $\Gamma$ is the set of solutions to (SCFP)-(1.1)), then $\left\{x_{n}\right\}$ converges weakly to a point $x^{*} \in \Gamma$.

(II) In addition, if $S$ is also semicompact, then $\left\{x_{n}\right\}$ and $\left\{u_{n}\right\}$ both converge strongly to $x^{*} \in \Gamma$.

Proof. The following is the proof of Theorem 3.1.

The Proof of Conclusion (I)

(1) First we prove that for each $\mathrm{p} \in \Gamma$, the following limits exist and

$$
\lim _{n \rightarrow \infty}\left\|x_{n}-p\right\|=\lim _{n \rightarrow \infty}\left\|u_{n}-p\right\|
$$

In fact, since $\phi$ is a continuous and increasing function, it results that $\phi(\lambda) \leq \phi(M)$, if $\lambda \leq M$, and $\phi(\lambda) \leq M^{*} \lambda^{2}$, if $\lambda \geq M$. In either case, we can obtain that

$$
\phi(\lambda) \leq \phi(M)+M^{*} \lambda^{2}, \quad \forall \lambda \geq 0 .
$$


Journal of Applied Mathematics

For any given $p \in \Gamma$, hence $p \in C:=F(S)$, and $A p \in Q:=F(T)$, from (3.1) and (2.7) we have

$$
\begin{aligned}
\left\|x_{n+1}-p\right\|^{2}= & \left\|u_{n}-p-\alpha_{n}\left(u_{n}-S^{n} u_{n}\right)\right\|^{2} \\
= & \left\|u_{n}-p\right\|^{2}-2 \alpha_{n}\left\langle u_{n}-p, u_{n}-S^{n} u_{n}\right\rangle+\alpha_{n}^{2}\left\|u_{n}-S^{n} u_{n}\right\|^{2} \\
\leq & \left\|u_{n}-p\right\|^{2}-\alpha_{n}(1-\beta)\left\|u_{n}-S^{n} u_{n}\right\|^{2} \\
& +\alpha_{n} \mu_{n} \phi\left(\left\|u_{n}-p\right\|\right)+\alpha_{n} \xi_{n}+\alpha_{n}^{2}\left\|u_{n}-S^{n} u_{n}\right\|^{2} \text { (by (2.7)) } \\
\leq & \left\|u_{n}-p\right\|^{2}-\alpha_{n}\left(1-\beta-\alpha_{n}\right)\left\|u_{n}-S^{n} u_{n}\right\|^{2} \\
& +\alpha_{n} \mu_{n}\left(\phi(M)+M^{*}\left(\left\|u_{n}-p\right\|\right)^{2}\right)+\alpha_{n} \xi_{n} \\
= & \left(1+\alpha_{n} \mu_{n} M^{*}\right)\left\|u_{n}-p\right\|^{2}-\alpha_{n}\left(1-\beta-\alpha_{n}\right)\left\|u_{n}-S^{n} u_{n}\right\|^{2} \\
& +\alpha_{n} \mu_{n} \phi(M)+\alpha_{n} \xi_{n} .
\end{aligned}
$$

On the other hand, since

$$
\begin{aligned}
\left\|u_{n}-p\right\|^{2} & =\left\|x_{n}-p+\gamma A^{*}\left(T^{n}-I\right) A x_{n}\right\|^{2} \\
& =\left\|x_{n}-p\right\|^{2}+\gamma^{2}\left\|A^{*}\left(T^{n}-I\right) A x_{n}\right\|^{2}+2 \gamma\left\langle x_{n}-p, A^{*}\left(T^{n}-I\right) A x_{n}\right\rangle,
\end{aligned}
$$

$$
\begin{aligned}
r^{2}\left\|A^{*}\left(T^{n}-I\right) A x_{n}\right\|^{2} & =\gamma^{2}\left\langle A^{*}\left(T^{n}-I\right) A x_{n}, A^{*}\left(T^{n}-I\right) A x_{n}\right\rangle \\
& =\gamma^{2}\left\langle A A^{*}\left(T^{n}-I\right) A x_{n},\left(T^{n}-I\right) A x_{n}\right\rangle \\
& \leq r^{2}\|A\|^{2}\left\|T^{n} A x_{n}-A x_{n}\right\|^{2}
\end{aligned}
$$

$$
\begin{aligned}
2 \gamma\left\langle x_{n}-p, A^{*}\left(T^{n}-I\right) A x_{n}\right\rangle & =2 \gamma\left\langle A x_{n}-A p,\left(T^{n}-I\right) A x_{n}\right\rangle \\
& \left.=2 \gamma\left\langle A x_{n}-A p\right)+\left(T^{n}-I\right) A x_{n}-\left(T^{n}-I\right) A x_{n},\left(T^{n}-I\right) A x_{n}\right\rangle \\
& =2 \gamma\left\{\left\langle T^{n} A x_{n}-A p, T^{n} A x_{n}-A x_{n}\right\rangle-\left\|\left(T^{n}-I\right) A x_{n}\right\|^{2}\right\} .
\end{aligned}
$$

In (2.8) taking $x=A x_{n}, G^{n}=T^{n}$, and $q=A p$ and noting $A p \in F(T)$, from (2.8) we have

$$
\begin{aligned}
& \left\langle T^{n} A x_{n}-A p, T^{n} A x_{n}-A x_{n}\right\rangle-\left\|\left(T^{n}-I\right) A x_{n}\right\|^{2} \\
& \quad \leq \frac{1+\kappa}{2}\left\|\left(T^{n}-I\right) A x_{n}\right\|^{2}+\frac{\mu_{n}}{2} \phi\left(\left\|A x_{n}-A p\right\|\right)+\frac{\xi_{n}}{2}-\left\|\left(T_{n}^{n}-I\right) A x_{n}\right\|^{2}
\end{aligned}
$$




$$
\begin{aligned}
& \leq \frac{\kappa-1}{2}\left\|\left(T^{n}-I\right) A x_{n}\right\|^{2}+\frac{\mu_{n}}{2}\left(\phi(M)+M^{*}\left\|A x_{n}-A p\right\|^{2}\right)+\frac{\xi_{n}}{2} \\
& \leq \frac{\kappa-1}{2}\left\|\left(T^{n}-I\right) A x_{n}\right\|^{2}+\frac{\mu_{n}}{2} M^{*}\|A\|^{2}\left\|x_{n}-p\right\|^{2}+\frac{\mu_{n}}{2} \phi(M)+\frac{\xi_{n}}{2} .
\end{aligned}
$$

Substituting (3.8) into (3.7), after simplifying it and then substituting the resultant result into (3.5), we have

$$
\begin{aligned}
\left\|u_{n}-p\right\|^{2} \leq & \left\|x_{n}-p\right\|^{2}+\gamma^{2}\|A\|^{2}\left\|T^{n} A x_{n}-A x_{n}\right\|^{2}+\gamma(\kappa-1)\left\|\left(T^{n}-I\right) A x_{n}\right\|^{2} \\
& +\gamma \mu_{n} M^{*}\|A\|^{2}\left\|x_{n}-p\right\|^{2}+\gamma \mu_{n} \phi(M)+\gamma \xi_{n} \\
= & \left\|x_{n}-p\right\|^{2}-\gamma\left(1-\kappa-\gamma\|A\|^{2}\right)\left\|T^{n} A x_{n}-A x_{n}\right\|^{2} \\
& +\gamma \mu_{n} M^{*}\|A\|^{2}\left\|x_{n}-p\right\|^{2}+\gamma \mu_{n} \phi(M)+\gamma \xi_{n} \\
\leq & \left(1+\gamma \mu_{n} M^{*}\|A\|^{2}\right)\left\|x_{n}-p\right\|^{2}-\gamma\left(1-\kappa-\gamma\|A\|^{2}\right)\left\|T^{n} A x_{n}-A x_{n}\right\|^{2} \\
& +\gamma \mu_{n} \phi(M)+\gamma \xi_{n} .
\end{aligned}
$$

Substituting (3.9) into (3.4) and simplifying it we have

$$
\begin{aligned}
\left\|x_{n+1}-p\right\|^{2} \leq & \left(1+\alpha_{n} \mu_{n} M^{*}\right)\left\{\left(1+\gamma \mu_{n} M^{*}\|A\|^{2}\right)\left\|x_{n}-p\right\|^{2}\right. \\
& \left.-\gamma\left(1-\kappa-\gamma\|A\|^{2}\right)\left\|T^{n} A x_{n}-A x_{n}\right\|^{2}+\gamma \mu_{n} \phi(M)+\gamma \xi_{n}\right\} \\
& -\alpha_{n}\left(1-\beta-\alpha_{n}\right)\left\|u_{n}-S^{n} u_{n}\right\|^{2}+\alpha_{n} \mu_{n} \phi(M)+\alpha_{n} \xi_{n} \\
= & \left(1+\delta_{n}\right)\left\|x_{n}-p\right\|^{2}-\gamma\left(1-\kappa-\gamma\|A\|^{2}\right)\left\|T^{n} A x_{n}-A x_{n}\right\|^{2} \\
& -\alpha_{n}\left(1-\beta-\alpha_{n}\right)\left\|u_{n}-S^{n} u_{n}\right\|^{2}+b_{n},
\end{aligned}
$$

where

$$
\begin{gathered}
\delta_{n}=\alpha_{n} \mu_{n} M^{*}+\gamma \mu_{n} M^{*}\|A\|^{2}+\gamma\|A\|^{2} \alpha_{n} \mu_{n}^{2}\left(M^{*}\right)^{2}, \\
b_{n}=\left(\left(1+\alpha_{n} \mu_{n} M^{*}\right) \gamma+\alpha_{n}\right) \mu_{n} \phi(M)+\left(\left(1+\alpha_{n} \mu_{n} \cdot M^{*}\right) \gamma+\alpha_{n}\right) \xi_{n} .
\end{gathered}
$$

By condition (iii) we have

$$
\left\|x_{n+1}-p\right\|^{2} \leq\left(1+\delta_{n}\right)\left\|x_{n}-p\right\|^{2}+b_{n}
$$


By condition (ii), $\sum_{n=1}^{\infty} \delta_{n}<\infty$ and $\sum_{n=1}^{\infty} b_{n}<\infty$. Hence it follows from Lemma 2.5 that the following limit exists:

$$
\lim _{n \rightarrow \infty}\left\|x_{n}-p\right\|
$$

Consequently, from (3.10) and (3.13) we have that

$$
\begin{aligned}
& r\left(1-\kappa-\gamma\|A\|^{2}\right)\left\|\left(T^{n}-I\right) A x_{n}\right\|^{2}+\alpha_{n}\left(1-\beta-\alpha_{n}\right)\left\|u_{n}-S^{n} u_{n}\right\|^{2} \\
& \quad \leq\left\|x_{n}-p\right\|^{2}-\left\|x_{n+1}-p\right\|^{2}+\delta_{n}\left\|x_{n}-p\right\|^{2} \longrightarrow 0 \quad(\text { as } n \longrightarrow \infty) .
\end{aligned}
$$

This together with the condition (iii) implies that

$$
\begin{gathered}
\lim _{n \rightarrow \infty}\left\|u_{n}-S^{n} u_{n}\right\|=0 \\
\lim _{n \rightarrow \infty}\left\|\left(T^{n}-I\right) A x_{n}\right\|=0 .
\end{gathered}
$$

It follows from (3.5), (3.13), and (3.16) that the $\operatorname{limit} \lim _{n \rightarrow \infty}\left\|u_{n}-p\right\|$ exists and

$$
\lim _{n \rightarrow \infty}\left\|u_{n}-p\right\|=\lim _{n \rightarrow \infty}\left\|x_{n}-p\right\|
$$

The conclusion (1) is proved.

(2) Next we prove that

$$
\lim _{n \rightarrow \infty}\left\|x_{n+1}-x_{n}\right\|=0, \quad \lim _{n \rightarrow \infty}\left\|u_{n+1}-u_{n}\right\|=0 .
$$

In fact, it follows from (3.1) that

$$
\begin{aligned}
\left\|x_{n+1}-x_{n}\right\| & =\left\|\left(1-\alpha_{n}\right) u_{n}+\alpha_{n} S^{n}\left(u_{n}\right)-x_{n}\right\| \\
& =\left\|\left(1-\alpha_{n}\right)\left(x_{n}+\gamma A^{*}\left(T^{n}-I\right) A x_{n}\right)+\alpha_{n} S^{n}\left(u_{n}\right)-x_{n}\right\| \\
& =\left\|\left(1-\alpha_{n}\right) \gamma A^{*}\left(T^{n}-I\right) A x_{n}+\alpha_{n}\left(S^{n}\left(u_{n}\right)-x_{n}\right)\right\| \\
& =\left\|\left(1-\alpha_{n}\right) \gamma A^{*}\left(T^{n}-I\right) A x_{n}+\alpha_{n}\left(S^{n}\left(u_{n}\right)-u_{n}\right)+\alpha_{n}\left(u_{n}-x_{n}\right)\right\| \\
& =\left\|\left(1-\alpha_{n}\right) \gamma A^{*}\left(T^{n}-I\right) A x_{n}+\alpha_{n}\left(S^{n}\left(u_{n}\right)-u_{n}\right)+\alpha_{n} \gamma A^{*}\left(T^{n}-I\right) A x_{n}\right\| \\
& =\left\|\gamma A^{*}\left(T^{n}-I\right) A x_{n}+\alpha_{n}\left(S^{n}\left(u_{n}\right)-u_{n}\right)\right\| .
\end{aligned}
$$

In view of (3.15) and (3.16) we have that

$$
\lim _{n \rightarrow \infty}\left\|x_{n+1}-x_{n}\right\|=0
$$


Similarly, it follows from (3.1), (3.16), and (3.20) that

$$
\begin{aligned}
\left\|u_{n+1}-u_{n}\right\|= & \left\|x_{n+1}+\gamma A^{*}\left(T^{n+1}-I\right) A x_{n+1}-\left(x_{n}+\gamma A^{*}\left(T^{n}-I\right) A x_{n}\right)\right\| \\
\leq & \left\|x_{n+1}-x_{n}\right\|+\gamma\left\|A^{*}\left(T^{n+1}-I\right) A x_{n+1}\right\| \\
& +\gamma\left\|A^{*}\left(T^{n}-I\right) A x_{n}\right\| \longrightarrow 0 \quad(\text { as } n \longrightarrow \infty) .
\end{aligned}
$$

The conclusion (3.18) is proved.

(3) Next we prove that

$$
\left\|u_{n}-S u_{n}\right\| \longrightarrow 0, \quad\left\|A x_{n}-T A x_{n}\right\| \longrightarrow 0 \quad(\text { as } n \longrightarrow \infty)
$$

In fact, from (3.15) we have

$$
\zeta_{n}:=\left\|u_{n}-S^{n} u_{n}\right\| \longrightarrow 0 \quad(\text { as } n \longrightarrow \infty)
$$

Since $S$ is uniformly L-Lipschitzian continuous, it follows from (3.18) and (3.23) that

$$
\begin{aligned}
\left\|u_{n}-S u_{n}\right\| & \leq\left\|u_{n}-S^{n} u_{n}\right\|+\left\|S^{n} u_{n}-S u_{n}\right\| \\
& \leq \zeta_{n}+L\left\|S^{n-1} u_{n}-u_{n}\right\| \\
& \leq \zeta_{n}+L\left\{\left\|S^{n-1} u_{n}-S^{n-1} u_{n-1}\right\|+\left\|S^{n-1} u_{n-1}-u_{n}\right\|\right\} \\
& \leq \zeta_{n}+L^{2}\left\|u_{n}-u_{n-1}\right\|+L\left\|S^{n-1} u_{n-1}-u_{n-1}+u_{n-1}-u_{n}\right\| \\
& \leq \zeta_{n}+L(1+L)\left\|u_{n}-u_{n-1}\right\|+L \zeta_{n-1} \longrightarrow 0 \quad(\text { as } n \longrightarrow \infty) .
\end{aligned}
$$

Similarly, from (3.16) we have

$$
\left\|A x_{n}-T^{n} A x_{n}\right\| \longrightarrow 0 \quad(\text { as } n \longrightarrow \infty)
$$

Since $T$ is uniformly $\widetilde{L}$-Lipschitzian continuous, by the same way as above, from (3.18) and (3.25), we can also prove that

$$
\left\|A x_{n}-T A x_{n}\right\| \longrightarrow 0 \quad(\text { as } n \longrightarrow \infty)
$$

(4) Finally we prove that $x_{n} \rightarrow x^{*}$ and $u_{n} \rightarrow x^{*}$ which is a solution of (SCFP)-(1.1).

Since $\left\{u_{n}\right\}$ is bounded, there exists a subsequence $\left\{u_{n_{i}}\right\} \subset\left\{u_{n}\right\}$ such that $u_{n_{i}} \rightarrow x^{*}$ (some point in $H_{1}$ ). From (3.22) we have

$$
\left\|u_{n_{i}}-S u_{n_{i}}\right\| \longrightarrow 0 \quad\left(\text { as } n_{i} \longrightarrow \infty\right)
$$

By Proposition 2.7, $S$ is demiclosed at zero; hence we know that $x^{*} \in F(S)$. 
Moreover, from (3.1) and (3.16) we have

$$
x_{n_{i}}=u_{n_{i}}-\gamma A^{*}\left(T^{n_{i}}-I\right) A x_{n_{i}} \rightarrow x^{*} .
$$

Since $A$ is a linear bounded operator, it gets $A x_{n_{i}} \rightarrow A x^{*}$. In view of (3.22) we have

$$
\left\|A x_{n_{i}}-T A x_{n_{i}}\right\| \longrightarrow 0 \quad\left(\text { as } n_{i} \longrightarrow \infty\right)
$$

Again by Proposition 2.7, $T$ is demiclosed at zero, and we have $A x^{*} \in F(T)$. Summing up the above argument, it shows that $x^{*} \in \Gamma$; that is, $x^{*}$ is a solution to the (SCFP)-(1.1).

Now we prove that $x_{n} \rightarrow x^{*}$ and $u_{n} \rightarrow x^{*}$.

Suppose to the contrary, if there exists another subsequence $\left\{u_{n_{j}}\right\} \subset\left\{u_{n}\right\}$ such that $u_{n_{j}} \rightarrow y^{*} \in \Gamma$ with $y^{*} \neq x^{*}$, then by virtue of (3.2) and the Opial property of Hilbert space, we have

$$
\begin{aligned}
\liminf _{n_{i} \rightarrow \infty}\left\|u_{n_{i}}-x^{*}\right\| & <\liminf _{n_{i} \rightarrow \infty}\left\|u_{n_{i}}-y^{*}\right\|=\lim _{n \rightarrow \infty}\left\|u_{n}-y^{*}\right\| \\
& =\lim _{n_{j} \rightarrow \infty}\left\|u_{n_{j}}-y^{*}\right\|<\liminf _{n_{j} \rightarrow \infty}\left\|u_{n_{j}}-x^{*}\right\| \\
& =\lim _{n \rightarrow \infty}\left\|u_{n}-x^{*}\right\|=\liminf _{n_{i} \rightarrow \infty}\left\|u_{n_{i}}-x^{*}\right\| .
\end{aligned}
$$

This is a contradiction. Therefore, $u_{n} \rightarrow x^{*}$. By using (3.1) and (3.16), we have

$$
x_{n}=u_{n}-\gamma A^{*}\left(T_{n}^{n}-I\right) A x_{n} \rightarrow x^{*} .
$$

The Proof of Conclusion (II)

By the assumption that $S$ is semicompact, it follows from (3.27) that there exists a subsequence of $\left\{u_{n_{i}}\right\}$ (without loss of generality, we still denote it by $\left\{u_{n_{i}}\right\}$ ) such that $u_{n_{i}} \rightarrow u^{*} \in H$ (some point in $H$ ). Since $u_{n_{i}} \rightarrow x^{*}$, this implies that $x^{*}=u^{*}$. And so $u_{n_{i}} \rightarrow x^{*} \in \Gamma$. By virtue of (3.2) we know that $\lim _{n \rightarrow \infty}\left\|u_{n}-x^{*}\right\|=0$ and $\lim _{n \rightarrow \infty}\left\|x_{n}-x^{*}\right\|=0$; that is, $\left\{u_{n}\right\}$ and $\left\{x_{n}\right\}$ both converge strongly to $x^{*} \in \Gamma$.

This completes the proof of Theorem 3.1.

The following theorem can be obtained from Theorem 3.1 immediately.

Theorem 3.2. Let $H_{1}$ and $H_{2}$ be two real Hilbert spaces, let $A: H_{1} \rightarrow H_{2}$ be a bounded linear operator, let $S: H_{1} \rightarrow H_{1}$ be a uniformly L-Lipschitzian and $\left(\beta,\left\{k_{n}^{(1)}\right\}\right)$-asymptotically strictly pseudocontractive mapping, and let $\mathrm{T}: \mathrm{H}_{2} \rightarrow \mathrm{H}_{2}$ be a uniformly $\widetilde{L}$-Lipschitzian and $\left(\kappa,\left\{k_{n}^{(2)}\right\}\right)$ asymptotically strictly pseudocontractive mapping satisfying the following conditions:

(i) $C:=F(S) \neq \emptyset, Q:=F(T) \neq \emptyset$;

(ii) $k_{n}=\max \left\{k_{n}^{(1)}, k_{n}^{(2)}\right\}$, and $\sum_{n=1}^{\infty}\left(k_{n}-1\right)<\infty$. 
Let $\left\{x_{n}\right\}$ be the sequence defined by (3.1), where $\left\{\alpha_{n}\right\}$ is a sequence in $[0,1]$ and $\gamma>0$ is a constant satisfying the following condition:

(iii) $\alpha_{n} \in(\delta, 1-\beta)$, for all $n \geq 1$ and $\gamma \in\left(0,(1-\kappa) /\|A\|^{2}\right)$, where $\delta \in(0,1-\beta)$ is a constant. If $\Gamma \neq \emptyset$, then the conclusions of Theorem 3.1 still hold.

From Theorems 3.1 and 3.2 we can obtain the following.

Theorem 3.3. Let $H_{1}$ and $H_{2}$ be two real Hilbert spaces, let $A: H_{1} \rightarrow H_{2}$ be a bounded linear operator, $S: H_{1} \rightarrow H_{1}$ be a uniformly L-Lipschitzian and $\beta$-strictly pseudocontractive mapping, and let $\mathrm{T}: \mathrm{H}_{2} \rightarrow \mathrm{H}_{2}$ be a uniformly $\widetilde{L}$-Lipschitzian and $\kappa$-strictly pseudocontractive mapping satisfying the following conditions:

(i) $C:=F(S) \neq \emptyset, Q:=F(T) \neq \emptyset$;

(ii) $T$ and $S$ both are demiclosed at origin.

Let $\left\{x_{n}\right\}$ be the sequence generated by

$$
\begin{gathered}
x_{1} \in H_{1} \text { chosen arbitrarily, } \\
x_{n+1}=\left(1-\alpha_{n}\right) u_{n}+\alpha_{n} S u_{n}, \\
u_{n}=x_{n}+\gamma A^{*}(T-I) A x_{n}, \quad \forall n \geq 1,
\end{gathered}
$$

where $\left\{\alpha_{n}\right\}$ is a sequence in $[0,1]$ and $\gamma>0$ is a constant satisfying the following condition:

(iii) $\alpha_{n} \in(\delta, 1-\beta)$, for all $n \geq 1$ and $\gamma \in\left(0,(1-\kappa) /\|A\|^{2}\right)$, where $\delta \in(0,1-\beta)$ is a constant. If $\Gamma \neq \emptyset$, then the conclusions of Theorem 3.1 still hold.

Proof. By the same way as given in the proof of Theorems 3.1 and 3.2 and noting that in the case of strictly pseudocontractive mapping the sequence $\left\{k_{n}=1\right\}$ in Theorem 3.2. Therefore we can prove that for each $p \in \Gamma$, the limits $\lim _{n \rightarrow \infty}\left\|x_{n}-p\right\|$ and $\lim _{n \rightarrow \infty}\left\|u_{n}-p\right\|$ exist and

$$
\begin{gathered}
\lim _{n \rightarrow \infty}\left\|x_{n}-p\right\|=\lim _{n \rightarrow \infty}\left\|u_{n}-p\right\| ; \\
\left\|u_{n}-S u_{n}\right\| \longrightarrow 0 ; \quad\left\|A x_{n}-T A x_{n}\right\| \longrightarrow 0 ; \\
\left\|u_{n}-u_{n+1}\right\| \longrightarrow 0 ; \quad\left\|x_{n}-x_{n+1}\right\| \longrightarrow 0 ; \\
x_{n} \longrightarrow x^{*} ; \quad u_{n} \rightarrow x^{*} \in \Gamma .
\end{gathered}
$$

In addition, if $S$ is also semicompact, we can also prove that $\left\{x_{n}\right\}$ and $\left\{u_{n}\right\}$ both converge strongly to $x^{*}$.

Remark 3.4. Theorems 3.1 and 3.2 improve and extend the corresponding results of Censor et al. [4, 5], Yang [7], Moudafi [9, 10], Xu [13], Censor and Segal [14], Masad and Reich [15], and others. 


\section{Acknowledgments}

The authors would like to express their thanks to the editors and the referees for their helpful comments and suggestions. This work was supported by the Natural Science Foundation of Yunnan Province and Yunnan University of Finance and Economics.

\section{References}

[1] Y. Censor and T. Elfving, "A multiprojection algorithm using Bregman projections in a product space," Numerical Algorithms, vol. 8, no. 2-4, pp. 221-239, 1994.

[2] C. Byrne, "Iterative oblique projection onto convex sets and the split feasibility problem," Inverse Problems, vol. 18, no. 2, pp. 441-453, 2002.

[3] Y. Censor, T. Bortfeld, B. Martin, and A. Trofimov, "A unified approach for inversion problem in intensity-modulated radiation therapy," Physics in Medicine E Biology, vol. 51, pp. 2353-2365, 2006.

[4] Y. Censor, T. Elfving, N. Kopf, and T. Bortfeld, "The multiple-sets split feasibility problem and its applications for inverse problems," Inverse Problems, vol. 21, no. 6, pp. 2071-2084, 2005.

[5] Y. Censor, A. Motova, and A. Segal, "Perturbed projections and subgradient projections for the multiple-sets split feasibility problem," Journal of Mathematical Analysis and Applications, vol. 327, no. 2, pp. 1244-1256, 2007.

[6] H.-K. Xu, "A variable Krasnosel'skii-Mann algorithm and the multiple-set split feasibility problem," Inverse Problems, vol. 22, no. 6, pp. 2021-2034, 2006.

[7] Q. Yang, "The relaxed CQ algorithm solving the split feasibility problem," Inverse Problems, vol. 20, no. 4, pp. 1261-1266, 2004.

[8] J. Zhao and Q. Yang, "Several solution methods for the split feasibility problem," Inverse Problems, vol. 21, no. 5, pp. 1791-1799, 2005.

[9] A. Moudafi, "The split common fixed-point problem for demicontractive mappings," Inverse Problems, vol. 26, no. 5, p. 055007, 2010.

[10] A. Moudafi, "A note on the split common fixed-point problem for quasi-nonexpansive operators," Nonlinear Analysis, vol. 74, no. 12, pp. 4083-4087, 2011.

[11] K. Aoyama, Y. Kimura, W. Takahashi, and M. Toyoda, "Approximation of common fixed points of a countable family of nonexpansive mappings in a Banach space," Nonlinear Analysis, vol. 67, no. 8, pp. 2350-2360, 2007.

[12] C. Martinez-Yanes and H.-K. Xu, "Strong convergence of the CQ method for fixed point iteration processes," Nonlinear Analysis, vol. 64, no. 11, pp. 2400-2411, 2006.

[13] H. K. Xu, "Iterative methods for the split feasibility problem in infinite-dimensional Hilbert spaces," Inverse Problems, vol. 26, no. 10, pp. 105018-17, 2010.

[14] Y. Censor and A. Segal, "The split common fixed point problem for directed operators," Journal of Convex Analysis, vol. 16, no. 2, pp. 587-600, 2009.

[15] E. Masad and S. Reich, "A note on the multiple-set split convex feasibility problem in Hilbert space," Journal of Nonlinear and Convex Analysis, vol. 8, no. 3, pp. 367-371, 2007. 


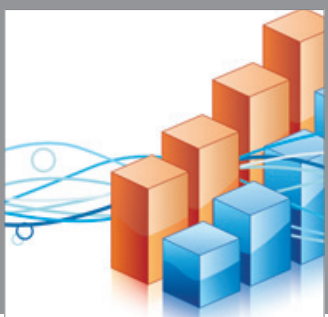

Advances in

Operations Research

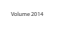

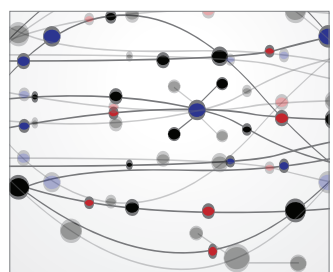

\section{The Scientific} World Journal
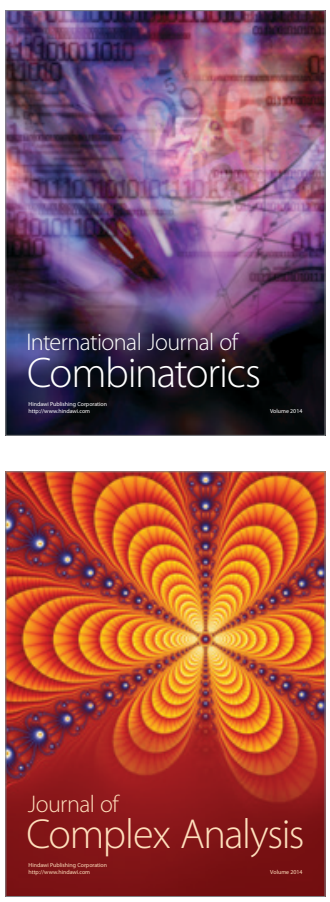

International Journal of

Mathematics and

Mathematical

Sciences
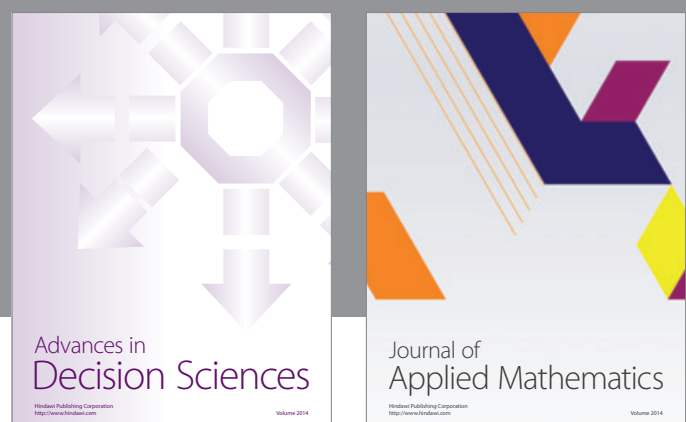

Journal of

Applied Mathematics
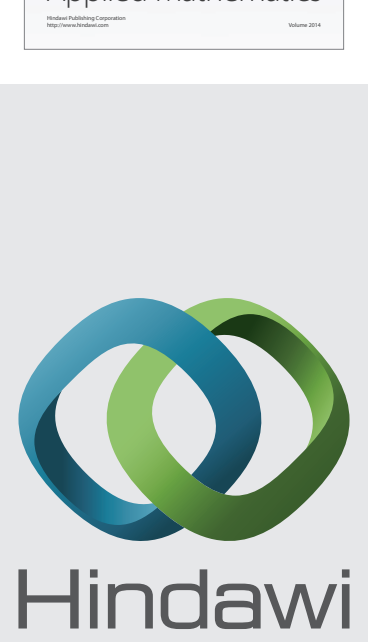

Submit your manuscripts at http://www.hindawi.com
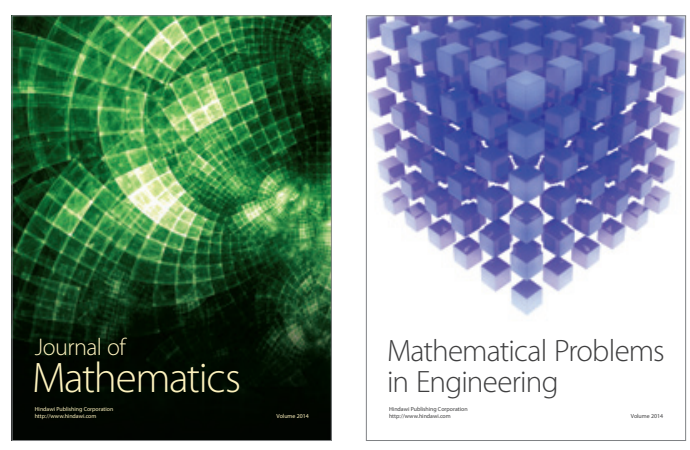

Mathematical Problems in Engineering
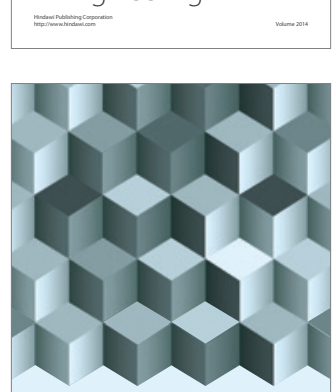

Journal of

Function Spaces
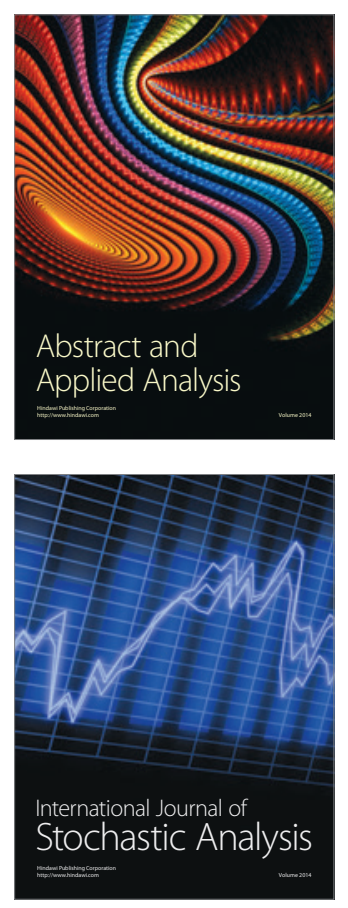

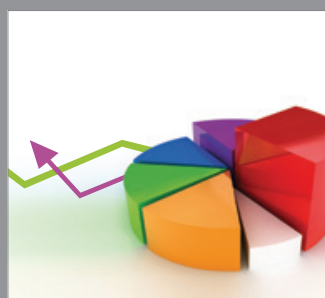

ournal of

Probability and Statistics

Promensencen
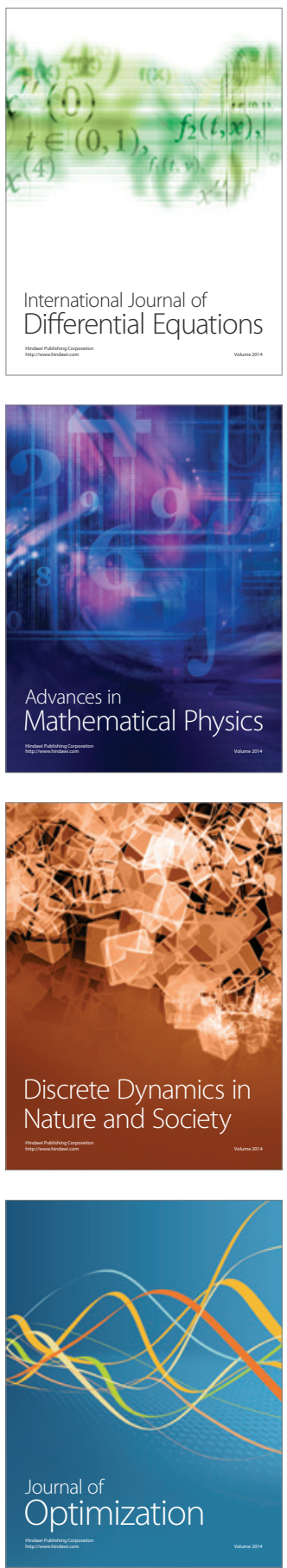